\title{
Tissue Culture of Normal and Cystic Fibrosis Sweat Gland Duct Cells I. Alterations in Dome Formation
}

\author{
DEBRA J. HAZEN-MARTIN, SAMUEL S. SPICER, MARY ANN SENS, MARGARET Q. JENKINS, \\ MILTON C. WESTPHAL, AND DONALD A. SENS \\ Departments of Pathology and Pediatrics, Medical University of South Carolina, \\ Charleston, South Carolina 29425
}

\begin{abstract}
The elucidation of the underlying defect in fluid secretion by cystic fibrosis (CF) sweat glands is hindered by the unavailability of an experimental model for investigating this disease. As a potential model system, a serum-free growth medium was developed that supports the explant growth of epithelial cells from fragments of human skin. Immunohistochemical analysis demonstrated that these epithelial cell outgrowths originated from the duct of the sweat gland. By electron microscopy, the cells were demonstrated to possess keratinocyte-like morphology as noted by the presence of a multilayered outgrowth of cells containing well-defined keratin bundles. Identical outgrowths from skin biopsies of $\mathrm{CF}$ patients were compared to normal outgrowths and alterations were noted to occur in dome formation and in the number of intercellular spaces between cells. Doming alterations were also noted to occur in the CF heterozygous state. No differences in cell fine structure or in growth factor requirements for cell proliferation were noted between normal and CF cells. The potential use of this system as a model for CF research is discussed. (Pediatr Res 21: 72-78, 1987)
\end{abstract}

\section{Abbreviations}

CF, cystic fibrosis

DME, Dulbecco's modified Eagles' medium

F-12, Ham's F-12 growth medium

PBS, phosphate-buffered saline

BrdU, bromodeoxyuridine

Recent studies by Quinton and coworkers (1-4), employing isolated sweat gland ducts from normal and CF volunteers, have demonstrated that the sodium transport mechanism in the CF sweat duct is as active as that in the normal duct. These studies also suggest that the decreased reabsorption of $\mathrm{NaCl}$ from $\mathrm{CF}$ sweat can be attributed largely, if not completely, to membrane impermeability to $\mathrm{Cl}$. Further studies have not demonstrated a factor in $C F$ sweat capable of altering the ion selective and transport properties of perfused human sweat ducts (5). These studies, therefore, strongly suggest that one of the manifestations of $C F$ results from a defect in the ability of the sweat gland duct to reabsorb chloride ion and that this defect may well reside in the sweat gland ductal epithelial cell itself. Whether $\mathrm{Cl}$ imperme-

Received January 15, 1986: accepted August 26, 1986.

Address for correspondence and reprints Donald A. Sens, Division of Cellular Biopathology, Department of Pathology, Medical University of South Carolina, 171 Ashley Avenuc, Charleston, SC 29425.

This work was supported by NIH Grant AM 11028. ability is the primary underlying defect in CF or one of several alterations of this disease state has not been conclusively demonstrated. To date, no alteration in the heterozygous state has been noted to occur nor has the underlying biochemical mechanism for $\mathrm{Cl}$ impermeability been defined.

The elucidation of the underlying defect in $\mathrm{CF}$, whether it be confined primarily to $\mathrm{Cl}$ ion impermeability or possibly other underlying mechanisms, is clearly hindered by the unavailability of an experimental model for investigating this disease. Without a model system, researchers have relied solely on normal and CF patient specimens for studies. Cultures composed of the epithelial cells that function abnormally in CF could provide such an experimental model, a role unsuccessfully attempted by fibroblast cultures $(6,7)$. No study to date has demonstrated the exact replicative origin of cultured sweat gland cells or differences between cells derived from normal and CF specimens. Thus, the goal of the present study was to establish primary cultures of human sweat duct epithelial cells from normal and CF skin, to determine the site of initial cell replication, and to provide preliminary evidence that the CF defect was expressed in these cultured epithelial cells.

\section{MATERIALS AND METHODS}

Reagents. DME, F-12, and fetal calf serum were obtained from Gibco Diagnostics (Grand Island, NY). Trypsin-EDTA $(0.05 \%$, $0.02 \%$ ) was obtained from Biofluids, Inc. (Rockville, MD). Components of the serum-free growth medium were obtained from Collaborative Research, Inc. (Lexington, MA). Bovine type I collagen was obtained from the Collagen Corp. (Palo Alto, CA). Reagents for the preparation of routine solutions were of reagent grade quality or higher and were obtained from Fisher Scientific (Atlanta, GA). Water for the preparation of all reagents was obtained from a MilliQ water system (Millipore Corp., Bedford, MA). Antiserum to human epidermal keratin was obtained from Dako Corporation (Santa Barbara, CA). Avidin-biotin kits for use in immunohistochemistry were obtained from Vector Labs (Burlingame, CA). Antisera to carbonic anhydrase isozymes were prepared in this laboratory and have been documented in a previous publication (8). Antiband 3 antibodies were obtained from Dr. Gary Pasternack and have been documented previously (9).

Specimen processing. In protocols defining the serum-free growth conditions of normal and CF sweat gland duct cells, human skin specimens were selected from samples removed less than $12 \mathrm{~h}$ postmortem. For protocols defining the dome formation differences between normal and CF state, human skin specimens were obtained by punch biopsy. Punch biopsies ( 5 $\mathrm{mm}$ ) were obtained from the deltoid region of the upper arm after thorough cleansing and Lidocaine injection of the area. The acquisition and use of human tissue was approved by the Internal 
Review Board for Human Research of the Medical University of South Carolina. Upon removal, the skin specimens were placed in sterile DME growth medium and transported to a biohazard hood in the cell culture facility where they were transferred to a $100-\mathrm{mm}$ sterile Petri dish containing DME growth medium. The skin specimens were dissected free of fat, if necessary, and cut with scissors and forceps into fragments of approximately $0.5 \mathrm{~mm}^{3}$. Six skin fragments were transferred into each of a series of $25 \mathrm{~cm}^{2}$ T-flasks containing a collagen type I/ adsorbed fetal calf serum matrix as described previously (10). The flasks were placed in an inverted position for $30 \mathrm{~min}$ at room temperature and then righted before adding $2.5 \mathrm{ml}$ of growth medium. The growth medium consisted of a $1: 1$ mixture of DME and F-12 supplemented with selenium $(5 \mathrm{ng} / \mathrm{ml})$, transferrin $(5 \mu \mathrm{g} / \mathrm{ml})$, insulin $(5 \mu \mathrm{g} / \mathrm{ml})$, hydrocortisone $(36 \mathrm{ng} / \mathrm{ml})$, triiodothyronine $(4 \mathrm{pg} / \mathrm{ml})$, prostaglandin $\mathrm{E}_{1}(10 \mathrm{ng} / \mathrm{ml})$, somatostatin $(10 \mathrm{ng} / \mathrm{ml})$, testosterone $(10 \mathrm{ng} / \mathrm{ml})$, dihydrotestosterone $(30 \mathrm{ng} / \mathrm{ml})$, and epidermal growth factor $(10 \mathrm{ng} / \mathrm{ml})$. The explants were incubated at $37^{\circ} \mathrm{C}$ in a $5 \% \mathrm{CO}_{2}-95 \%$ air atmosphere and left undisturbed for 3 days. The cultures were fed fresh growth medium every 3 days until confluent.

Examination of cultures. Growth of viable cultures was monitored by examination at intervals with an Olympus IM inverted microscope. Photomicrographs of cultures were taken on $35 \mathrm{~mm}$ Kodak Panatomic X film. A record of the morphological characteristics of the viable cells in growing and confluent cultures was maintained by development of contact sheets. The photographic documentation of the domes was obtained by dark field microscopy.

Normal and $\mathrm{CF}$ cultures initiated from tissue acquired at postmortem examination were fixed for light and electron microscopic examination at approximately weekly intervals starting 7 days after explantation. Cultures obtained by biopsy were fixed for light and electron microscopy after 33 days in culture following the quantitation of dome formation. Explant fragments and outgrowths were fixed in situ with $2.5 \%$ glutaraldehyde in $0.1 \mathrm{M}$ $\mathrm{PBS}, \mathrm{pH} 7.4$, for $0.5 \mathrm{~h}$ at room temperature. The outgrowths were postfixed with $2 \%$ osmium tetroxide in $0.1 \mathrm{M}$ PBS for 0.5 $\mathrm{h}$ at room temperature and dehydrated in a series of alcohols. The flasks were then filled with Epon 812. Culture flasks were then removed from the polymerized Epon. Enface and transverse sections were obtained from reembedded portions of outgrowth. Thick sections were stained with toluidine blue for light microscopic viewing and thin sections from selected blocks were stained with uranyl acetate-lead citrate and examined in a JEOL $100 \mathrm{~S}$ electron microscope. Two to five outgrowth areas from each explant were examined.

Permeability of cell layers in the outgrowth of the skin cultures was tested using lanthanum tracer technique described by Revel and Karnovsky (11). Modifications were made for use of the procedure in a culture system. Growth medium was drained from confluent cultures and the contents of the culture vessel were rinsed twice with physiological saline for a total rinse time of $10 \mathrm{~min}$. The cultures were then fixed in situ with $2.5 \%$

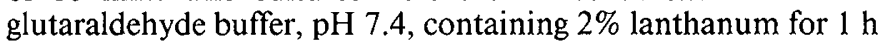
at room temperature. Following fixation the cultures were rinsed with $0.1 \mathrm{M}$ cacodylate buffer three times for a total rinse time of $10 \mathrm{~min}$. The cultures were then postfixed for $2 \mathrm{~h}$ at room temperature with a solution of $1 \%$ osmium tetroxide in $0.1 \mathrm{M}$ cacodylate buffer containing $2 \%$ lanthanum and routinely embedded in Epon 812 for ultrastructural analysis. Thin sections were examined without uranyl acetate-lead citrate staining.

Quantitation of dome formation. Cultures derived from the normal and CF patient skin biopsies were allowed to grow for 33 days and photogram prints were obtained by placing the culture flasks directly on a high contrast photographic paper (Kodachrome IIRC) and exposing. The resulting photograms, or contact prints, allow visualization of doming areas as dark circles with white rims. The leading edge of the culture outgrowths were outlined as well. For each flask, the total area of cell outgrowth, the number of domes, and the area of each dome were quanti- tated utilizing a Zeiss Videoplan image analysis system. The resulting data were then expressed as the percent of the total cell outgrowth occupied by areas of dome formation for cultures derived from normal, $\mathrm{CF}$ heterozygote, and $\mathrm{CF}$ homozygote patient skin specimens. Student's $t$ test was utilized for the assignment of significance between groups. The normal volunteers, CF heterozygotes, and CF homozygotes were all caucasians and were not matched for age or sex. Only females were utilized in the CF heterozygote group.

Quantitation of intercellular spaces. In addition to qualitative assessment of intercellular spaces, one set of normal and CF 25day duct cell outgrowth thick sections were stained with toluidine blue and further analyzed with a Zeiss Videoplan image analysis system. Over 400 oil immersion $(\times 1000)$ fields were examined for each duct cell outgrowth and representative areas of each outgrowth were examined. The parameters quantitated included the number of viable cells per field, the number of intercellular spaces per field, and the size of the intercellular spaces per field.

Immunohistochemistry. The avidin-biotin system was utilized for the immunohistochemical demonstration of epidermal keratin, band 3 protein, carbonic anhydrase, and smooth muscle myosin (12). Explant fragments were removed from the culture flasks for examination at day 1 through 14 and were fixed for 12 $\mathrm{h}$ in Carnoy's fluid and transferred to $100 \%$ ethanol. The tissue was cleared in xylene and paraffin-embedded. Sections of the explant fragment, $5 \mu$ thick, were deparaffinized and rehydrated prior to staining. Controls included staining of serial sections with nonimmune serum and staining of sections of Carnoy-fixed human skin derived from samples used for culture. Sites of cell proliferation in 1- to 7-day old skin explant fragments were determined by the addition of BrdU to the culture system. BrdU was added to achieve a final concentration of $10 \mu \mathrm{M} 12 \mathrm{~h}$ prior to the removal of fragments for fixation and processing for immunocytochemistry as described above. Cells which incorporated BrdU, a thymidine analogue, into DNA were then visualized by immunostaining using monoclonal antibody to $\mathrm{BrdU}$ (Anti-BrdU, Becton Dickinson, Mountain View, CA).

\section{RESULTS}

Explant culture of human sweat gland epithelial cells. Utilizing the described growth medium, cell growth from fragments of human skin was observed between 3-7 days after culture initiation. Phase contrast microscopy revealed that after 7 days of active growth, cell outgrowths were composed of uniform cuboidal cells that appeared as a monolayer (Fig. 1 $a$ ). After an additional 14 days of proliferation, cultures were confluent and cells were observed in uneven multilayers (Fig. 1b). Between 22 and 33 days in culture, cells acquired a squamous appearance (Fig. 1c). Further outward proliferation of cells from the explant fragments was very minimal at this stage.

Histochemical identification as sweat gland duct cells. A series of immunocytochemical localizations revealed both the site of cell proliferation in explant fragments as well as the immunocytochemical profile of the proliferating cells (Table 1). BrdUlabeled explant fragments identified the sweat duct as the origin of cell proliferation. At day 1 of culture, each sweat coil contained several labeled sweat duct nuclei. At days 2 and 3, increased numbers of labeled duct nuclei were observed and by day 4 (Fig. $2 a$ ), each sweat gland coil contained as many as 40 labeled duct cell nuclei. Labeled nuclei were not observed in any other component of the skin explant fragment at any time interval examined, effectively removing these elements from consideration as proliferative centers in the explant fragment. Of particular significance was the total absence of labeled nuclei in the region of the epidermis (Fig. $2 b$ ).

Extensive keratin-positive cell proliferation was observed in the coiled portion of the sweat duct (Fig. $2 c$ ). The sweat duct, normally composed of two cell layers, became multilayered while keratin-positive myoepithelial cells as well as secretory coil cells appeared to degenerate in culture. Proliferating keratin-positive duct cells (Fig. $2 d$ ) reached the cut surfaces of the dermis and 

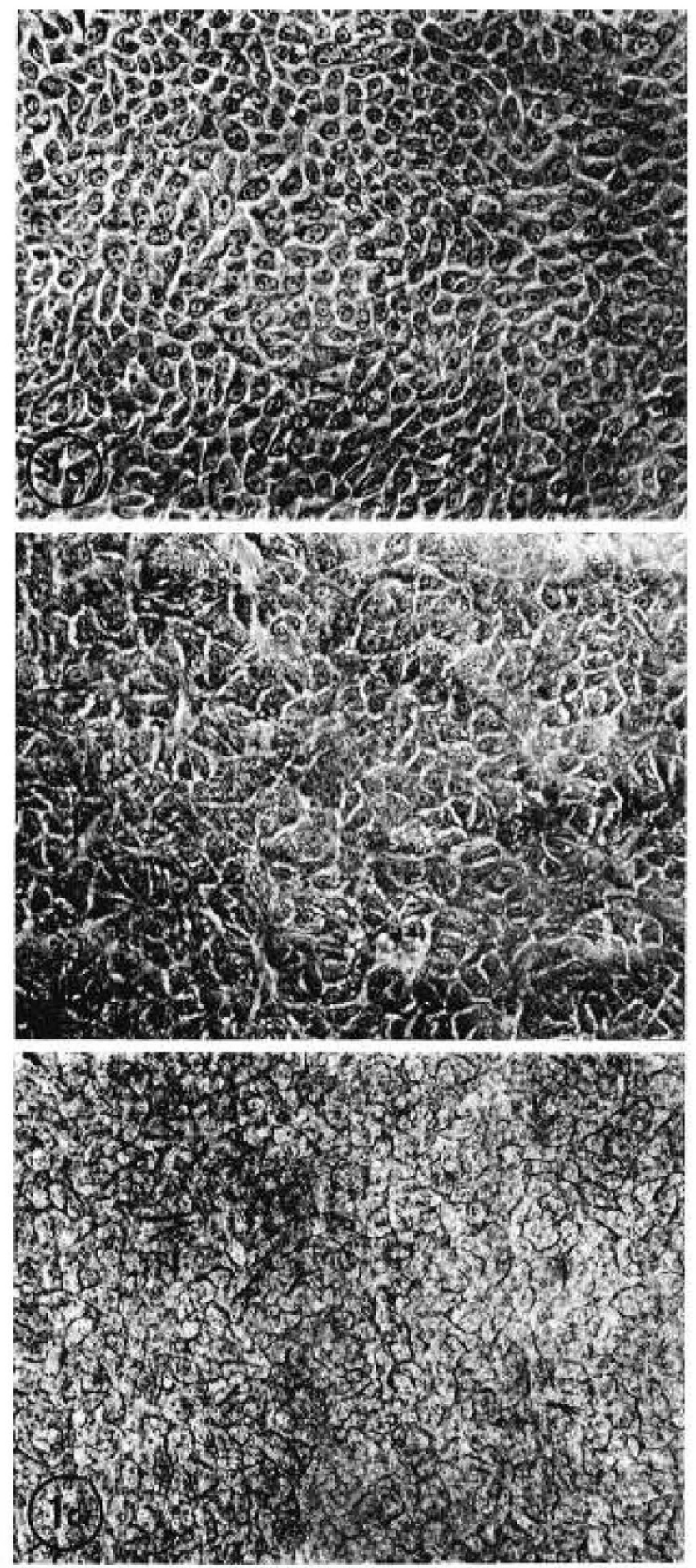

Fig. 1. Epithelial cell outgrowth from normal human skin specimens after: $a, 7$ days of growth, $b, 21$ days of growth, and $c, 33$ days of growth, $\times 100$.

Table 1 . In vivo and in vitro immunocytochemical profiles

\begin{tabular}{lcccc}
\hline & $\begin{array}{c}\text { Proliferating } \\
\text { cells* } \\
\text { in vitro }\end{array}$ & $\begin{array}{c}\text { Sweat duct } \\
\text { in vivo }\end{array}$ & $\begin{array}{c}\text { Secretory } \\
\text { coil } \\
\text { in vivo }\end{array}$ & $\begin{array}{c}\text { Myoepithelial } \\
\text { cells } \\
\text { in vivo }\end{array}$ \\
\hline Myosin & - & - & - & + \\
Keratin & + & + & \pm & + \\
CA III & + & + & - & - \\
Band 3 & + & + & - & - \\
\hline
\end{tabular}

* Cells identified by BrdU labeling both within and growing over the surface of the explant fragment. grew over the surface of the explant fragment to reach the culture vessel surface. In addition, carbonic anhydrase III as well as band 3 (Fig. 2e) immunoreactivity, characteristic of in vivo sweat duct, was observed in proliferating cells both within and on the surface of the explant fragment. The proliferating cells were clearly myosin-negative, further ruling out proliferation of keratin-positive myoepithelial cells in this culture system.

Explant culture of $C F$ sweat gland duct cells. Skin specimens obtained from two CF postmortem examinations were assessed for their ability to produce primary explant cultures of sweat gland duct cells. The specimens were placed into culture under identical conditions to that described previously for normal skin specimens and were also tested for any alterations in growth factor requirements through a deletion study. No differences in light microscopic morphology, ability to explant, degree of ker-
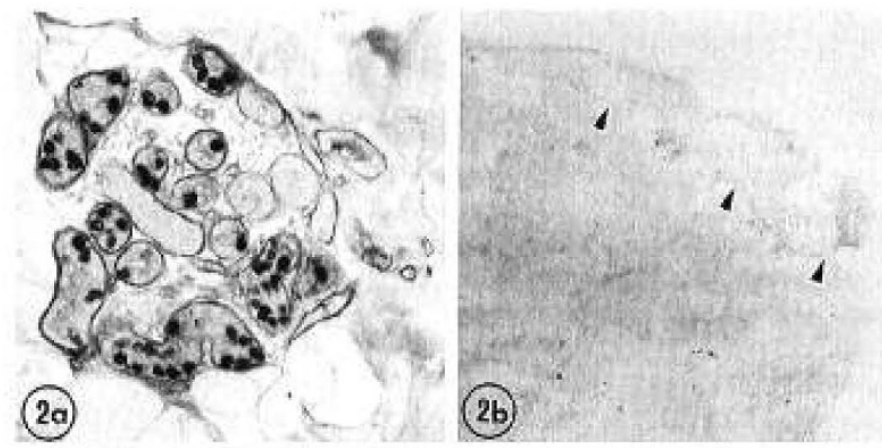

(2c)
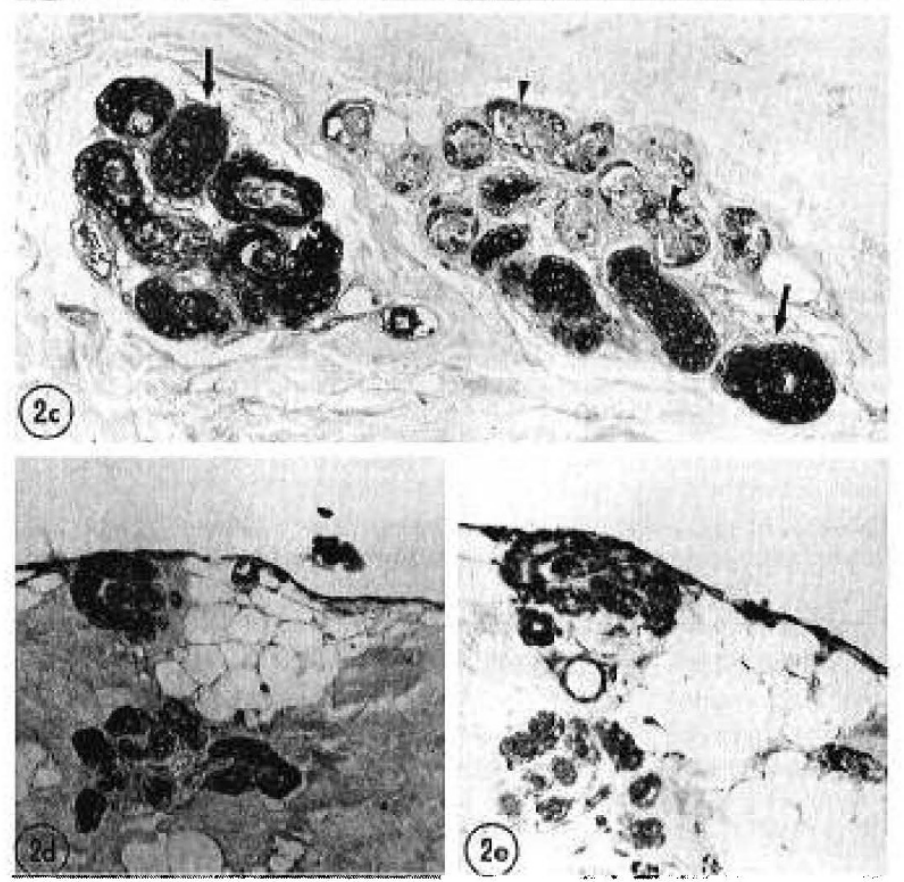

Fig. 2. $a$, day 4 normal BrdU-labeled explant, anti-BrdU with Alcian blue-PAS counterstain. Nuclei of ducts within the coiled segment of the sweat gland represent the only site of BrdU incorporation thereby confirming the ductal origin of cell proliferation in this tissue, $\times 250$. $b$, day 3 normal BrdU-labeled explant, anti-BrdU. The lack of BrdU label at the epidermal-dermal junction (arrowheads) seen in this fragment was representative of each time period observed, $\times 140$. $c$, day 5 normal explant, antikeratin. Proliferating duct cells in the sweat coil region stain intensely for keratin (arrows). Remnants of keratin-stained myoepithelial cells (arrowheads) surround degenerating secretory regions of the coil, $\times 280$. $d$, day 7 normal explant, antikeratin. Proliferating cells reaching the exterior dermal surface of the fragment are immunoreactive for keratin, $\times 160$. $e$, day 7 normal explant antiband 3 protein. Cells on the surface of the explant fragment are immunoreactive for this duct-specific antigen, $\times 160$. 
atinization, or culture life span were observed in CF cultures compared with normal cultures. Results of the deletion studies failed to disclose any alteration in growth factor requirements from those defined for growth of the normal cells.

There was, however, one obvious difference between normal and CF cells in culture. This difference involved what is best described as "macrodome" production by the isolated CF sweat gland duct cells. The classic production of domes in cell culture involves the focal accumulation of fluid between the cell monolayer (or multilayer) and the growth surface of the culture flask. The requirements for dome formation are believed to be the presence of active ion transport by the cells as well as tight junctions between cells and attachment of the cells to the culture growth surface (13). Classic domes are microscopic and require magnification of approximately $\times 100$ for visualization. Domes produced by CF sweat gland duct cells were defined as "macrodomes" due to their large size and were visible without the aid of magnification (Fig. $3 a$ ). Dark field microscopy, without magnification, demonstrated the clear difference in macrodome formation between CF sweat gland duct cells and those isolated from non-CF skin specimens (Fig. $3 b$ and $c$ ).

The appearance of CF macrodomes was closely correlated with terminal differentiation of the culture. Dome formation did not occur during the period of active outward cell growth from the explant fragment. As determined by light microscopic examination, domes first appeared as large, slightly out of focus areas once the uppermost layer of the culture was beginning to keratinize. Thereafter, the domes increased only slightly in diameter, but increased rapidly in height until the doming area was clearly visible without the aid of microscopic evaluation. Once formed, the domes were permanent in nature without collapse and reformation in other areas of the culture flask. The general appearance of the macrodomes resembled a blister on the heel of the foot following irritation.

Differences in macrodome formation between normal and $C F$ sweat gland duct cells. With the suggestion of a qualitative difference in dome formation in cultures of normal versus $C F$ human sweat gland duct cells, a skin biopsy protocol was initiated. For this study, skin biopsies were obtained from 15 normal volunteers, seven CF heterozygotes, and $12 \mathrm{CF}$ homozygotes. The skin biopsies from all three groups were obtained and placed into cell culture under identical conditions. After 33 days in culture, the number and area of the domes were quantitated and the results expressed as the percent of the total area of cell outgrowth occupied by domes. Clear differences in macrodome formation were observed between normal, $\mathrm{CF}$ homozygous, and heterozygous cultures (Table 2). The normal mean $(8.2 \pm 2.1)$ when compared to that of either the CF heterozygous (14.8 \pm $3.8)$ or homozygous state $(17.7 \pm 4.5)$ was significant at $p<$ 0.001 . The $C F$ heterozygous state compared to the homozygous state demonstrated no significant difference $(p>0.05)$. This analysis strongly suggested that the underlying defect of $C F$ could be expressed in cultures initiated from both the $\mathrm{CF}$ heterozygote and homozygote state.

Controls performed for the above study were limited in scope since the size of the biopsy obtained from each individual was sufficient to initiate only a single explant flask from most of the samples. Variability in macrodome formation between multiple isolated samples collected at the same time from a single individual and repeatability of macrodome formation for a given individual were not tested. These studies were not performed since patient benefit from multiple biopsies could not be justified. Within this limitation, several parameters were analyzed and suggested that the samples obtained yielded valid results. The area of cell growth for the normal, heterozygote, and homozygote cultures were approximately equal which demonstrated that macrodome formation was not influenced by unequal cell growth. The ages and sex of the CF patients and volunteers used in the biopsy protocol had no significant influence on macrodome formation. Lastly, the number of macrodomes formed by
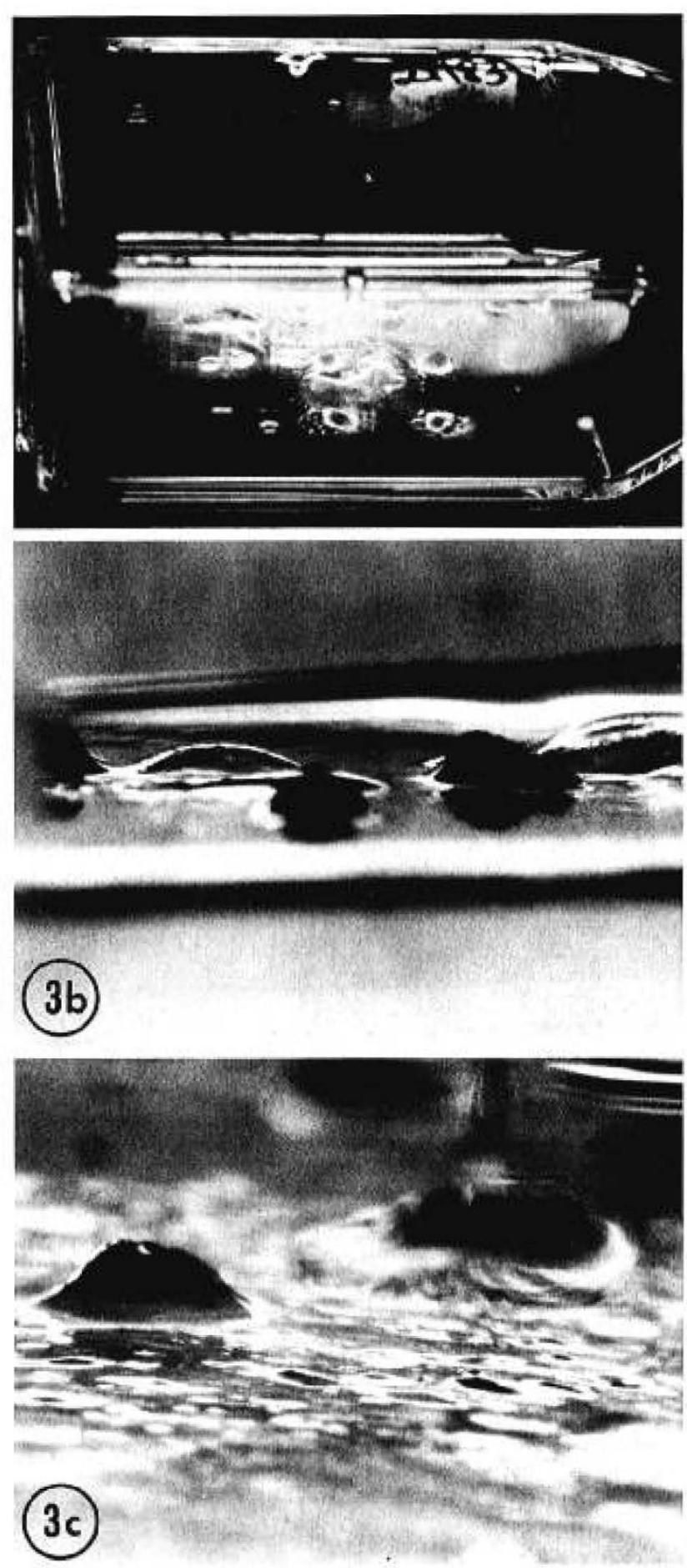

Fig. 3. Macrodome formation by cultured sweat gland duct cells. $a$, a 33-day CF culture, $\times 1.3 . b$, dark field microscopy of a 32 day $\mathrm{CF}$ culture, $\times 5.0$. $c$, dark field microscopy of a 33-day normal culture, $\times 7.0$.

Table 2. Macrodome formation by normal and CF sweat gland duct cells

Patient

$\%$ Cell outgrowth

occupied by

domes $($ mean \pm SEM)

$\begin{array}{lr}\text { Normals }(n=15) & 8.2 \pm 2.1 \\ \text { Heterozygote }(n=7) & 14.8 \pm 3.8 \\ \text { Homozygote }(n=12) & 17.7 \pm 4.5\end{array}$


each individual culture was not significantly different in the normal, heterozygote, and homozygote groupings.

Ultrastructural characteristics. With the exception of intercellular spaces between cells, outgrowths from skin explants from both normal and CF subjects showed no qualitative differences in fine structure when comparable regions of outgrowth and cultures of similar age were compared. Cells in the outgrowth were observed in a multilayered arrangement with the most viable cells located in the bottom cell layers closest to the culture flask surface (Fig. 4).

The most basal cells possessed a rounded nucleus with a single nucleolus and contained numerous polysomes (Fig. 4). Profiles of granular reticulum, a supra- or paranuclear Golgi complex (Fig. 5), and fairly numerous mitochondria were observed. These cells characteristically exhibited numerous bundles of tonofilaments which often formed a network encircling the nucleus and

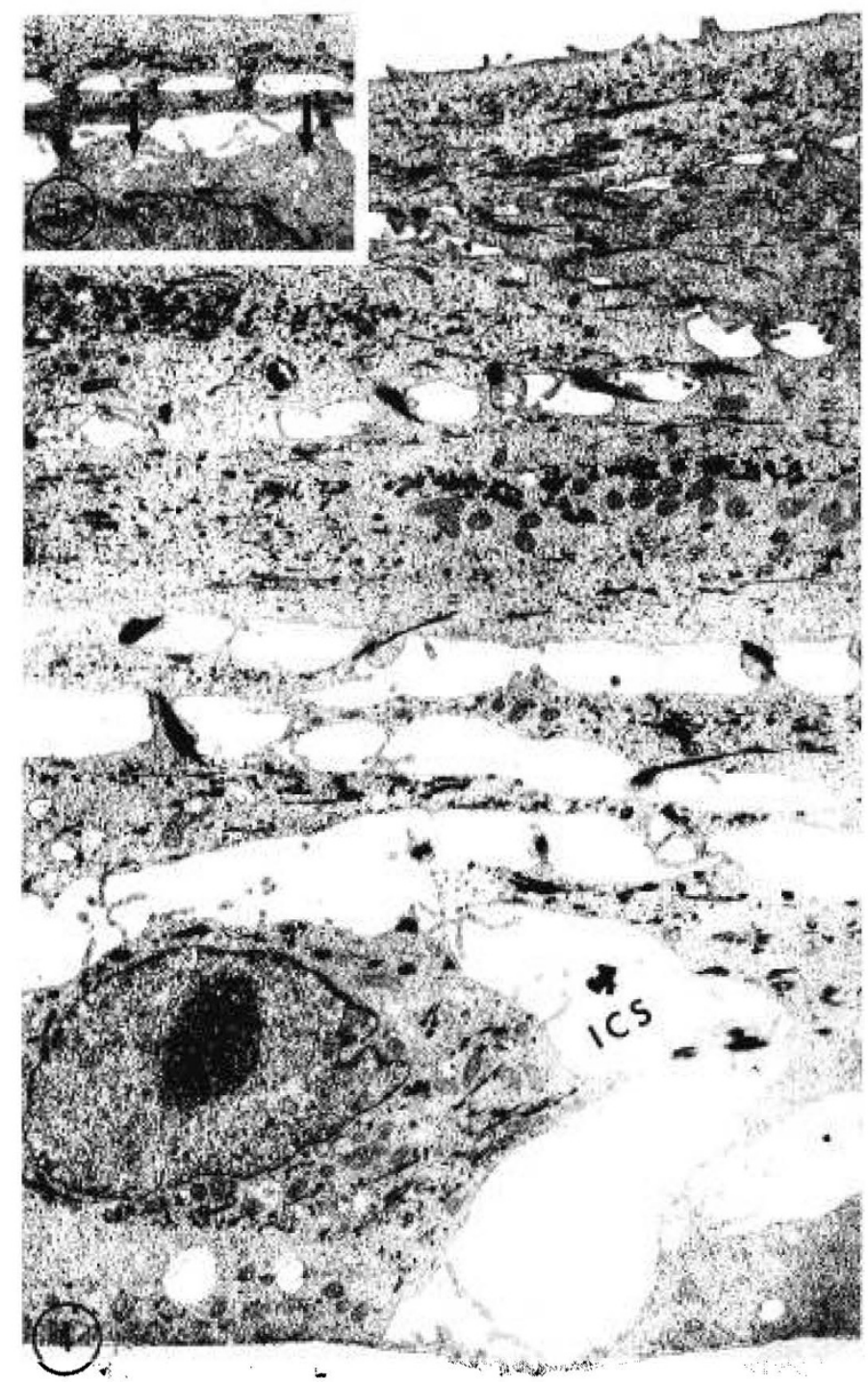

Fig. 4. CF skin outgrowth. A multilayered area of outgrowth displays a progression of changes toward the surface, including flattening of cells and loss of nuclei and mitochondria. Wide intercellular space (ICS) separate basal and second layer cells, but decrease toward the surface. Cuboidal basal cell contains an ovoid nucleus with a large nucleolus, $\times 7500$.

Fig. 5. CF skin outgrowth. The supranuclear area of a basal cell shows prominent Golgi complexes (arrows) and tonofilaments terminating in desmosomes at microvilli contacts between adjacent cells, $\times 6900$. extended to desmosomal connections with neighboring cells at the tips of microvilli which transversed prominent intercellular spaces (Figs. 4 and 5). Dilated intercellular spaces were highly variable in number and size; however, the general observation was that there were more spaces in normal than in CF-derived cultures.

Cultures exposed to the tracer, lanthanum, revealed electronopaque material in intercellular spaces between the top one to three cell layers but not below (Fig. 6). Intercellular spaces between the bottom three layers of cells failed to reveal the dense tracer material, thereby confirming the intact nature of these spaces.

Differences in intercellular spaces of CF and normal duct cells. Examination of toluidine blue-stained thick sections served as a method for inspecting differences in intercellular spaces between outgrowths from normal and CF patients. Visual observation of duct cell outgrowths from 25- to 31-day cultures derived from five normal and five CF patients yielded the general conclusion that these intercellular spaces were far more prevalent in cultures derived from normal individuals than those from $C F$ individuals. The results of the more detailed Videoplan analysis demonstrated that there was no difference in the viability or number of cells present on the surface of the culture vessel of the normal versus $\mathrm{CF}$ duct cell outgrowths. However, the number of intercellular spaces was different between normal and CF duct cell outgrowths with CF-derived cultures exhibiting fewer intercellular spaces between adjacent cells (Fig. 7). In contrast, the areas of individual intercellular spaces, when present, were not different between normal and CF duct cell outgrowths.
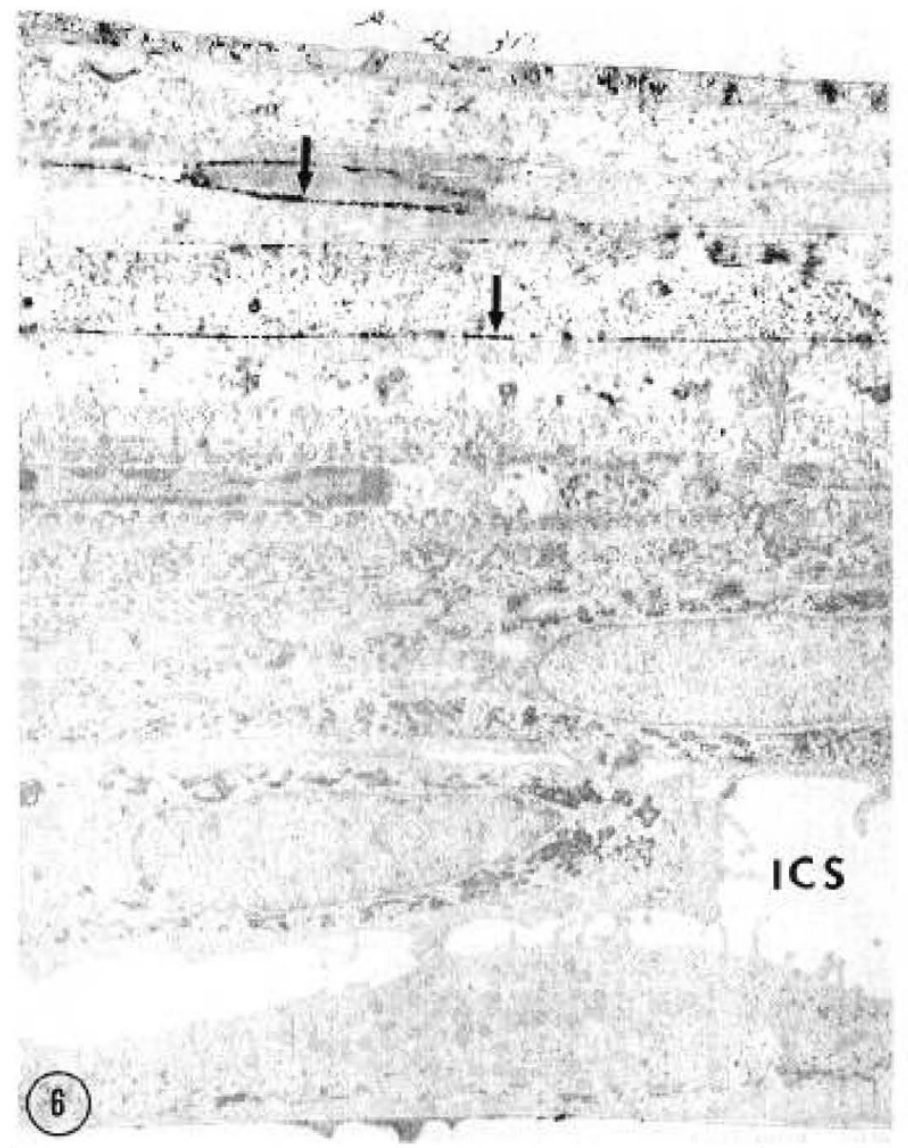

Fig. 6. A 20-day normal duct cell culture exposed to lanthanum during fixation. Note that the tracer penetrates the upper two to three cell layers (arrows). Tracer material was never observed in the dilated intercellular spaces $(I C S)$ in the basal half of the outgrowth, $\times 7700$. 


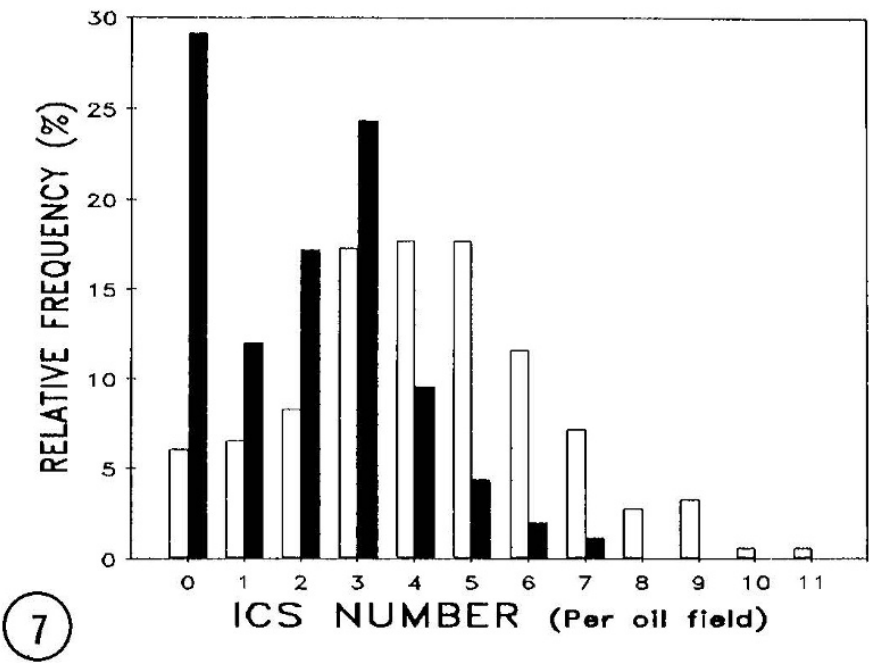

Fig. 7. The number of intercellular spaces in normal (open bars) and $\mathrm{CF}$ duct cell outgrowths (closed bars). The relative frequency represents the percent occurrence in the total number of fields.

\section{DISCUSSION}

Sweat glands are the most consistently affected but least damaged organs involved in CF (14). For this reason, the sweat gland is an excellent source of viable tissue on which to base an epithelial cell tissue culture model for $\mathrm{CF}$ research. This choice is further reinforced by current studies revealing an insensitivity of the secretory portion of the gland to $\beta$-adrenergic stimulation (15) and defective ion transport in the reabsorptive duct of the CF sweat gland (3). Clearly, the development of sweat glandderived epithelial cell cultures represents a logical initial step in the formation of a cell culture model for CF research.

Explanted fragments of human skin were utilized for the initiation of cultures of sweat gland epithelial cells over other techniques such as dissection of the glands and enzymatic tissue digestion for two reasons. First, the opportunity to obtain large amounts of $\mathrm{CF}$ tissue is rare and the use of explanted fragments of skin allows the rapid initiation of many cultures in a short period of time. More than 150 cultures of sweat gland epithelial cells may be initiated in a single workday. Second, and of central importance, is that the utilization of an explant technique preserved the tissue for the analysis of the site of origin of cell proliferation within the tissue. This was clearly demonstrated by the identification of cell replication in the ductal segment of the sweat gland and subsequent immunochemical analysis of these cells. The opportunity for identification of cell origin is lost when dissection or enzymatic dispersion of tissue occurs.

Several lines of evidence suggested that the sweat gland duct cells retained in vivo properties in culture. These included the demonstration of keratin, carbonic anhydrase III, and band 3 protein, all shown to occur in the sweat gland duct in vivo in the proliferating cells. These cells were also distinguished from epidermal keratinocytes by the observation that the multilayered duct cells lacked the keratohyaline characteristic of terminal differentiation and cornification occurring in stratified squamous epithelium $(16,17)$. Other features of the sweat gland duct cells which suggested differentiated function included dome formation and the microscopic demonstration of dilated space between cells in the bottom two layers of the cultures. Classically, dome formation is thought to result from active ion transport, attachment of the cells to the culture surface, and the presence of occluding junctions between cells (13). Sweat gland duct cells exhibited all of these properties except tight junctions between adjacent cells, raising the question as to the "tightness" of the multilayer for fluid retention. The inability of lanthanum to penetrate lower intercellular spaces clearly confirmed the ability of the multilayer to retain accumulated fluid. The mechanism underlying fluid retention by these cell multilayers, in the absence of tight junctions, is analogous to the permeability barrier provided, in vivo, by the keratinocytes comprising the human skin. Human skin provides a permeability barrier in the absence of tight junctions, and the most common example of this function is blister formation caused by chronic rubbing of the skin surface. The mechanism underlying fluid retention by human skin is not totally clear, but presumably involves the deposition of lipid between intercellular spaces of keratinocytes which effectively blocks fluid flow (see Reference 18 for review). Since the sweat gland duct cells described herein present in culture as keratinocyte-like (resembling human skin keratinocytes), their ability to retain fluid and form macrodomes is not surprising. In fact, the cell membranes of the terminally differentiated layers of the sweat duct cell cultures on ultrastructural examination do demonstrate a strong osmiophilic staining pattern indicative of the presence of lipid. Presumably, macrodomes are not noted in skin keratinocyte cultures due to a lack of inherent transport activity, whereas the duct cell cultures retain and express this known in vivo function. Similarly, the dilated intercellular space between adjacent cells in the bottom two layers of cells was also interpreted and evidence of active secretion of fluid and ions by basal cells. The only alternate explanation would be formation of an artificial space due to fixation artifact. This artifact, however, is effectively removed from consideration by results of the lanthanum tracer study. The tracer, included in the fixative solution, would have penetrated the basal cell intercellular spaces if the spaces had formed during the fixation process itself. These results, taken together, provide ample evidence for the retainment of differentiated function by these cells.

Results of the biopsy study comparing sweat gland duct cells of normal and $\mathrm{CF}$ individuals can be summarized as follows. 1) There was no difference in the growth medium requirements between the normal and $\mathrm{CF}$ state. 2) There was no readily observable difference in the culture lifespan, morphology, or fine structural detail between the normal and CF state. 3) There was a significant difference in the ability of the normal and CF cells to form macrodomes with both heterozygote and homozygote samples exceeding normals in the ability to form domes. 4) The number of intercellular spaces appeared decreased in CF homozygote cultures compared to normal cultures. Increased macrodome formation in CF-derived sweat gland duct cell cultures appears inconsistent with defective $\mathrm{Cl}$ reabsorption. While no precise explanation can be given for this discrepancy, one hypothesis is offered. It is entirely plausible that the defect in $\mathrm{Cl}$ reabsorption, noted to occur in CF sweat gland ducts in situ, involves an alteration in the cell membrane. A general type of alteration need not be limited in effect to a single parameter such as $\mathrm{Cl}$ transport, but could also be expressed in vitro as altered attachment of the cells to the culture vessel growth surface, one of the three parameters affecting dome formation by cells in culture. Therefore, a weakness in the attachment of CF cells to the culture matrix could lead to an increase in size of domes regardless of $\mathrm{Cl}$ impermeability. In culture, $\mathrm{Cl}$ ion could be free to follow a paracellular route leading to subsequent fluid retention and macrodome formation.

In conclusion, the initiation of pure sweat duct cultures, combined with evidence for a difference between the normal and $\mathrm{CF}$ state, identify this culture system as a logical first step in the continuing development of optimum conditions for an epithelial cell culture model for $\mathrm{CF}$ research.

Acknowledgments. The authors thank Ms. Martha Rudisill and Ms. Dorothea Hintz for excellent technical assistance and Ms. Sandy Varner for excellent editorial assistance. The antibody to smooth muscle myosin was a gift from Dr. Mark Willingham at the National Institutes of Health. 


\section{REFERENCES}

1. Bijman J, Quinton PM 1984 Abnormal electrolyte transport in cystic fibrosis sweat ducts as a function of $\mathrm{Cl}$ impermeability. Am J Physiol 247:C3-C9

2. Quinton PM 1981 Effects of some transport inhibitors on secretion and reabsorption in intact and perfused single human sweat glands. Pflug Arch Eur J Physiol 391:309-313

3. Quinton PM 1983 Chloride impermeability in cystic fibrosis. Nature 301:421422

4. Quinton PM, Bijman J 1983 High bioelectric potential due to decreased chloride absorption in the sweat glands of patients with cystic fibrosis. $\mathrm{N}$ Engl J Med 308:1185-1189

5. Bijman J, Quinton PM 1984 Apparent absence of cystic fibrosis sweat factor on ion-selective and transport properties of the perfused human sweat duct. Pediatr Res 18:1292-1296

6. Collie G. Buchwald M. Harper P. Riordan JR 1985 Culture of sweat gland epithelial cells from normal individuals and patients with cystic fibrosis. In Vitro 21:597-602

7. Pederson PS 1984 Primary culture of epithelial cells derived from the reabsorptive coiled duct of human sweat glands. IRCS Med Sci 12:752-753

8. Spicer SS, Sens MA. Hennigar RH. Stoward PJ 1984 Implications of the immunohistochemical localization of the carbonic anhydrase isoenzymes for their function in normal and pathological cells. Ann NY Acad Sci 429:382397

9. Hazen-Martin DJ, Pasternack G, Spicer SS. Sens DA 1986 Immunocytochem- ical localization of band 3 protein in normal and cystic fibrosis skin. $\mathbf{J}$ Histochem Cytochem 34:823-826

10. Detrisac CJ, Sens MA, Garvin AJ, Spicer SS, Sens DA 1984 Tissue culture of human kidney epithelial cells of proximal tubule origin. Kidney Int 25:383390

11. Revel JP, Karnovsky MJ 1967 Hexagonal array of subunits in intercellular junctions of the mouse heart and liver. J Cell Biol 33:C7-Cl6

12. Hsu SM, Raine L 1981 Avidin and biotin in immunohistochemistry. J Histochem Cytochem 19:1349-1353

13. Cereijido M, Ehrenfeld J, Fernandez-Castelo S, Meza I 1981 Fluxes, junctions and blisters in cultured monolayers of epithelioid cells (MDCK). Ann NY Acad Sci 372:422-441

14. Cage GW, Dobson RL, Waller R 1966 Sweat gland function in cystic fibrosis. $\mathrm{J}$ Clin Invest 45:1373-1380

15. Sato K, Sato F 1985 Defective beta-adrenergic response of cystic fibrosis sweat glands in vivo and in vitro. $\mathrm{J}$ Clin Invest 73:1763-1771

16. Green H 1979 The keratinocyte as differentiated cell type. Harvey Lect 84:101139

17. Rheinwald JG, Green H 1975 Serial cultivation of strains of human epidermal keratinocytes: the formation of keratinizing colonies from single cells. Cell $6: 331-344$

18. Scheuplein RJ, Bronaugh RL 1983 Percutaneous absorption. In: Goldsmith LA (ed) Biochemistry and Physiology of the Skin, Vol II. Oxford University Press, New York, pp 1255-1302 